\title{
REFORÇO E RECOMPENSA: A GAMIFICAÇÃO TRATADA SOB UMA ABORDAGEM BEHAVIORISTA
}

Reinforcement and reward: The gamification treated under a behaviorist approach

MENEZES, Graciela Sardo I graegra@gmail.com

TARACHUCKY, Laryssa I It_lara@yahoo.com.br

PELLIZZONI, Roger Costa I roger@pellizzoni.com.br

PERASSI, Richard L. I richard.perassi@uol.com.br

GONÇALVES, Marília Matos I marilinhamt@gmail.com

GOMEZ, Luiz Salomão Ribas I salodesigner@gmail.com

FIALHO, Francisco Antonio Pereira I fapfialho@gmail.com

Universidade Federal de Santa Catarina - UFSC

\begin{abstract}
Resumo
Este artigo apresenta o conceito de gamificação e explora a contribuição da ciência do comportamento expressa no behaviorismo radical para a criação de envolvimento com o usuário. São apresentados dados bibliográficos que justificam e conceituam o termo gamificação e sua relação com o behaviorismo radical de Skinner. Sistemas gamificados são sugeridos como ferramenta capazes de auxiliar na mudança de conduta, individuais ou coletivas, visando o reforço de comportamentos considerados positivos para a sociedade.

Palavras-chave: Gamificação. Behaviorismo. Condicionamento operante.

\section{Abstract}

This article introduces the concept of gamification and explores the contribution of the philosophy of science of behavior expressed in radical behaviorism to the process that creates involvement with the user. Bibliographic data are presented that justify and conceptualize the term gamification and its relationship with the radical behaviorism of Skinner. The gamified systems are presented as tool that can assist in changing behavior, both individual and collective, aiming at reinforcing behaviors considered positive for society.

Keywords: Gamification. Behaviorism. Operant conditioning.
\end{abstract}




\section{INTRODUCCÃO}

Pensar a gamificação sem explorar os processos mentais que ocorrem nas pessoas envolvidas com atividades gamificadas pode tornar sua abordagem prejudicialmente superficial. O behaviorismo radical apresenta notáveis contribuições para o uso do pensamento e da mecânica de jogos em outros contextos na medida em que delineia uma concepção de comportamento (o comportamento operante), facilitando no entendimento da mecânica dos processos de envolvimento do usuário.

De acordo com Sridharan, Hrishikesh e Raj (2012), a gamificação é o uso do pensamento e da mecânica dos jogos em outros contextos a fim de envolver os usuários e resolver problemas. Ela promove motivação e pode encorajar pessoas a participar de algo. Suas técnicas são usadas em aplicações e processos para melhorar o envolvimento dos usuários, aumentar o retorno sobre o investimento, a qualidade dos dados, a percepção de oportunidades e elevar o nível de aprendizado.

A satisfação, de acordo com a teoria behaviorista de Skinner (1982) é um reforço do comportamento na medida em que ela afeta a probabilidade de um organismo responder a determinado estímulo de maneira a alcançar uma consequência reforçadora. O ser humano, como um ser lúdico (HUIZINGA, 1980), encontra satisfação nos jogos, especialmente como entretenimento.

O objetivo deste artigo é expor através de uma abordagem qualitativa a relação entre o behaviorismo radical e a gamificação, explorando os conceitos de cada área e as contribuições do primeiro para o desenvolvimento de atividades gamificadas que promovam efetivo engajamento dos participantes. Para tanto, os dados coletados são apresentados em duas sessões. A primeira apresenta os dados bibliográficos que justificam e conceituam o termo gamificação, enquanto a segunda expõe sua relação com o behaviorismo radical de Skinner (1982).

\section{GAMIFICAC̄̃O: UM CONCEITO EM PROCESSO}

Assim como acontece com outros conceitos relacionados à área de estudos hipermidiáticos, pesquisas relacionadas à gamificação são recentes e, ainda, escassas. O próprio termo somente veio a ser mencionado pela primeira vez no início do ano 2002, por Nick Pelling quando da descrição de seu trabalho como consultor de marketing. Entretanto, a expressão e o significado a ele atrelado não receberam a atenção, seja do universo acadêmico ou empresarial, até o segundo semestre de 2010, quando alguns players do segmento da indústria de jogos e eventos científicos da área proporcionaram que o conceito viesse a ser exposto ao grande público, ocupando, a partir daí, um crescente espaço. 
Assim que a indústria de games interessou-se pelo assunto, diversos produtos gamificados foram desenvolvidos para diferentes usos e fins, extrapolando a previsível aplicação de entretenimento e abrangendo desde as áreas da saúde, educação e sustentabilidade, até projetos para o ambiente de gestão de negócios, visando o aumento da produtividade, racionalização das finanças, gestão de equipes e ações de marketing e comunicação (XU, 2012).

Com a crescente aplicação de suas técnicas em âmbito comercial, e a sua natural exposição, deu-se início a estudos mais aprofundados a respeito. As poucas definições vinculadas ao termo 'gamificação' originam-se do conceito inicial formulado por Deterding et al. (2011a), que a considera como "o uso de elementos de design de jogos em contextos de não-jogos". O ambiente empresarial, do qual se originou a gamificação, contudo, não parece fazer uma distinção tão clara entre estes contextos. Segundo Azarite (2013, p. 16-17) "a gamification se da em qualquer momento em que ha algum tipo de ludicidade ou competitividade envolvendo a realização de determinada tarefa."

Neste sentido, seu significado e aplicação amplia-se para formatos os mais diversificados, desde que estes apresentem regras pré-estabelecidas a serem seguidas e, em contrapartida, ofereçam em troca alguma espécie de estímulo positivo como motivação e satisfação aos usuários que cumpram os objetivos do "jogo", ou, pelo menos, àqueles que alcancem determinado destaque em relação aos demais participantes envolvidos. Vale ressaltar, contudo, que a gamificação não é, ou está, obrigatoriamente vinculada à criação de um jogo ou brincadeira 'premiável'. Qualquer proposta que possua caráter lúdico ou de desafio pode ser considerada como uma ação gamificada (AZARITE, 2013).

Entretanto, a visão mercadológica da gamificação tem entre suas premissas um interesse comercial. Neste sentido, para o marketing, a gamificação seria uma técnica que faz uso da ferramenta de um jogo visando atender às estratégias de uma empresa, marca ou produto. Para Azarite (2013, p. 6) a gamificação está, obrigatoriamente, atrelada a algum objetivo de negócio: uma pesquisa de mercado, uma aproximação maior com seu público-alvo ou, ainda, a geração de crowdsourcing, modelo de produção que utiliza a inteligência e os conhecimentos coletivos e voluntários para resolver problemas, criar conteúdo e soluções ou desenvolver novas tecnologias, assim como também para gerar fluxo de informação.

A gamification se mostra uma estratégia útil para condicionar o comportamento humano numa infinidade de situações. A apropriação disso para negócios é clara: gamification para estimular usuários, clientes, colaboradores ou prospects a se engajarem em torno de algum objetivo específico (AZARITE, 2013, p. 16). 
Entre as representações mais utilizadas de gamificação em âmbito e interesse empresarial estão (1) a criação de "barras de progresso" para manter um perfil atualizado num e-commerce ou rede social; (2) sentimento de exclusividade ou de um status diferenciado para aqueles que colaborarem com a empresa naquilo que for necessário (crowdsourcing); (3) jogos e outras formas lúdicas para a realização de treinamentos para colaboradores; e (4) retratos espalhados pela empresa para os "funcionários do mês".

A abordagem científica, entretanto, considera fundamental que as implicações relacionadas ao conceito da gamificação não se restrinjam ao ambiente corporativo e mercadológico. Segundo Groh (2012) é preciso que, em primeiro lugar, se proceda a uma distinção clara entre os termos que dão origem e sustentação ao conceito da gamificação. "É preciso distinguir entre "game" (a palavra em inglês para 'jogo') e "play" (o verbo 'jogar' em inglês). Tendo em conta que a palavra "play" é empregada tanto para jogos quanto para brincadeiras, vale ressaltar que há uma diferença substancial entre ambas. Seguindo o conceito de Caillois (2006), que ao descrever os termos paidia (jogo), como uma forma livre e expressiva de comportamentos improvisados e significativos, e ludus (jogo) como caracterizado por um jogo baseado em regras com determinados objetivos, estabelece-se aí uma clara distinção, já em sua origem etimológica, entre ambas as expressões. Nesse sentido, gamificação, como versão de "ludificação", estaria mais identificada com os elementos do design de jogos (ou seja, ludus), sendo a parte relacionada à diversão um aspecto que pode ou não estar vinculado à mecânica do produto gamificado. Neste sentido, há concordância da visão mercadológica na conclusão de Azarite (2013, p. 29) ao dizer que "a gamification não é obrigatoriamente a criação de um jogo ou de uma brincadeira para seu cliente", uma proposta que contemple alguns aspectos de ludicidade e com uma abordagem desafiadora atende de forma similar às prerrogativas de um produto gamificado.

Encontramos aí, então, um ponto de convergência conceitual entre o meio acadêmico e o industrial, indicando ser o foco da gamification quase que exclusivamente em ludus, com apenas um pouco de espaço para paidia $(G R O H$, 2012).

\section{GAMIFICAC̣ÃO EM CONTEXTOS DE NÃO-GAMES}

Elementos vinculados ao design de jogos possuem, normalmente, propósitos de entretenimento. Contudo, o uso de tais elementos em produtos gamificados serve, em princípio, com o objetivo de tornar menos estressante ou tedioso o processo em contextos não-games, os chamados jogos sérios. Deterding et. al. (2011b) ressalta, contudo, que ainda não foi possível concluir a real vantagem em determinar-se uma distinção clara entre contextos de 
games e não-games. Isso poderia precipitar uma avaliação superficial da real conceituação da gamificação, se voltada para um objetivo 'sério' ou apenas por 'diversão'. De qualquer modo, a ideia de contextos não-games, ou de 'jogos sérios' gira em torno da aplicação da gamificação em atividades ligadas ao treinamento de equipes, de geração de informação e notícias, de produtos e serviços voltados à saúde, à educação, entre outras áreas de aplicação.

Como mostrado na Figura 1, a definição de Deterding et. al. separa gamificação de jogos sérios e de design de interação lúdica. Enquanto o eixo vertical separa jogos (gaming) de jogos (playing), respectivamente 'jogos sérios' de 'gamificação', o eixo horizontal distingue 'gamificação' (design gameful) de design lúdico, bem como de brinquedos, se ambos os eixos forem considerados.

Com a gamificação aspira-se a apropriação de elementos de jogos a outros contextos, não o comportamento ou atitude lúdica. Esta diferença é importante na medida em que os jogos são estruturas para o comportamento humano definidas por regras, enquanto que a brincadeira ou o comportamento lúdico são expressões do comportamento humano (FRASCA, 1999). Os autores estão conscientes que a criação de estruturas de comportamento baseadas em princípios ou elementos de jogos pode potencializar indiretamente $\mathrm{O}$ comportamento lúdico. No entanto, o enfoque está nos jogos como estruturas para ação com regras e objetivos. O desenvolvimento de atividades utilizando elementos de games insere o componente lúdico em atividades necessárias como agente de promoção da satisfação do usuário. A motivação através de gamificação pode potencialmente modificar algum comportamento e criar hábitos, estimular pessoas ao engajamento de atividades "sérias" e alterar a experiência vivenciada por elas em relação a um determinado contexto.

Figura 01 - Separação do termo 'gamificação' frente a jogos 'sérios' em partes/ todo no eixo, bem como do design 'divertido' paralelamente ao eixo de jogo/jogar.

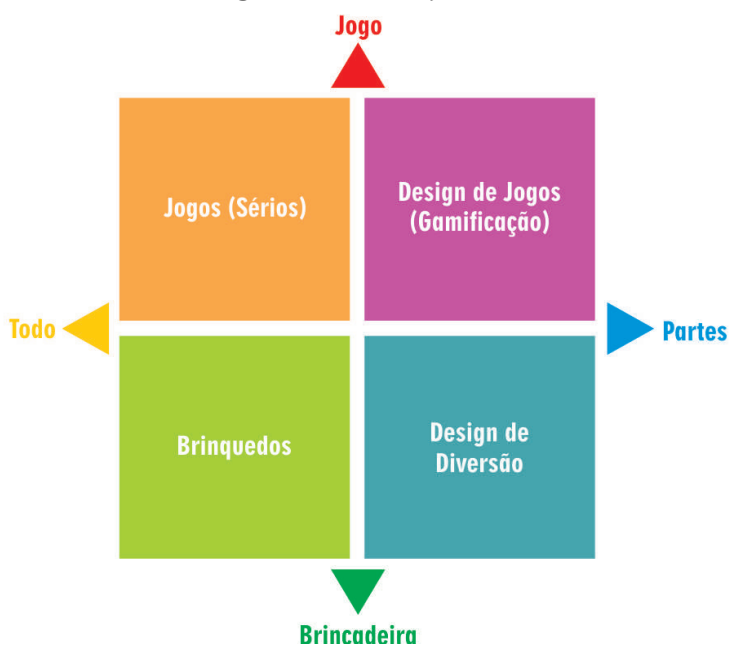

Fonte: Adaptado de Groh (2012, p. 39). 
Um game é composto por uma série de escolhas significativas, e permite o jogador a permanecer em uma zona de conforto segregada do mundo real. Os games oferecem experiências sociais que podem ser transmitidas para o mundo real e, frequentemente, jogar um game modifica certos aspectos do comportamento humano. De acordo com Bakkes, Tan e Pisan (2012), quando as pessoas jogam games, eles estão batendo em suas melhores qualidades, a sua capacidade de ser motivados, para ser otimista, para colaborar com os outros, para ser resiliente em face da falha (BAKKES; TAN; PISAN, 2012). As habilidades aprendidas durante os jogos precisam ser aproveitadas para o bem social (MOORE, 2011).

\section{REFORÇO E RECOMPENSA COMO CONDICIONANTES DO COMPORTAMENTO EM ATIVIDADES GAMIFICADAS}

Behaviorismo radical é a filosofia da ciência do comportamento, na qual é delineada uma concepção do comportamento (o comportamento operante). $O$ behaviorismo radical afirma que a natureza dota o indivíduo de certas motivações e que, ao longo do seu desenvolvimento, a interação com o ambiente confere outras motivações a ele. Os estímulos oferecidos pelo ambiente suscitam respostas que, por sua vez, serão recompensadas ou punidas, reforçando ou reduzindo determinados comportamentos. Assim, o indivíduo aprende a repetir certos atos, enquanto "desaprende" outros, moldando, aos poucos, um conjunto de comportamentos que, somados, constituem uma espécie de repertório da sua conduta frente ao mundo (ZILIO, 2010). Por meio da análise experimental, várias ocorrências comportamentais são observadas e postas em relação com variáveis ambientais, sendo que, a partir dessa análise, é possível observar a ocorrência de mudanças ordenadas, e, assim, o delineamento de padrões de comportamento.

Comportamento, segundo Skinner (1982), é a parte do funcionamento do organismo responsável pela sua ação sobre, ou interação com, o mundo externo, ou então, "a relação essencial, pressuposta e contínua entre o ambiente e as ações de um organismo." (ZILIO, 2010, p. 73). Pressupõe-se que o comportamento seja ordenado, no sentido de ser regido por leis, e que, consequentemente, seja determinado, no sentido de ocorrer em função de eventos passados. De acordo com a teoria behaviorista, um comportamento é fortalecido pelas consequências que ele produz e por esta razão, as próprias consequências são chamadas de "reforços". Portanto, a base do processo de condicionamento operante por reforço positivo reside na ideia de que resultados satisfatórios aumentam a chance de ocorrência do comportamento que o produziu. Reforço é a consequência de um comportamento que se mostra capaz de alterar a frequência deste, tornando-o mais ou menos provável. O reforço positivo ocorre quando um comportamento é seguido de 
um estímulo recompensador. Um reforço positivo aumenta a probabilidade de um comportamento pela presença de uma recompensa (ZILIO, 2010). Muitos reforços favoráveis ocorrem internamente e os chamados esquemas da razão fornecem alguns exemplos: 1) zelo, diligência ou ambição; 2) determinação, obstinação, persistência ou perseverança; 3) excitação ou entusiasmo; 4) dedicação ou compulsão (SKINNER, 1982).

Skinner (1982) declarou que o homem é um ser sensível a um reforçamento positivo, movido pela satisfação, pelo prazer e pelas consequências da sua ação. Se as consequências de uma ação forem punitivas ele cessa o comportamento até chegar numa supressão chamada "supressão do responder". Sendo positivas, a tendência é haver o reenforçamento desta atividade. Tal visão pode parecer simplista a uma primeira leitura, remetendo ao conceito estereotipado do behaviorismo, relacionando-o exclusivamente ao modelo Pavloviano. Porém, levando-se em conta que satisfação envolve aspectos complexos da natureza humana, passa a haver a necessidade de uma discussão mais profunda sobre as recompensas resultantes em reforços positivos.

A satisfação é um reforço para o comportamento humano, relacionandose com a saciação, que é um efeito dos reforçadores (SKINNER, 1982). A palavra "satisfação" deriva do Latin satis (suficiente) e facere (fazer). Ela pode ser considerada sob duas dimensões essenciais: a satisfação referente a uma experiência específica e a satisfação acumulada. $O$ indivíduo aprende com as experiências passadas, modificando seu comportamento e expectativas com relação a experiências futuras. A satisfação do indivíduo pode causar uma série de consequências sobre o seu comportamento com relação às reações que ele tem e com que frequência ele tem essas reações. Oliver (2010) elenca cinco estados emocionais que favorecem o aumento da satisfação: aceitação, alegria, alívio, interesse/excitação e prazer.

O princípio hedônico sugere que as pessoas buscam alcançar o prazer e evitar a dor e que esta motivação básica é subjacente a todas as outras. Todos os organismos nascem com algumas motivações e adquirem outras através da experiência.

Ballone (2008) afirma que as pessoas estão entediadas. Com isso, descreve uma condição comunmente observada no desenvolvimento de tarefas obrigatórias do dia a dia. Seja pelo que ele chama de "falta de volição/ vontade" de fazer ou pela sensação de "falta de capacidade" para fazer, a falta de motivação para a realização de determinadas tarefas não é algo facilmente alterável.

Estudos apresentados por Kohn (1999) demonstraram que quando 
crianças são pagas para desenhar tendem a desenhar mais, porém, com menos qualidade e sua satisfação por desenhar diminui. A este é dado o nome "overjustification" que descreve quando a motivação intrínseca alterna para a extrínseca. Por motivação extrínseca entendem-se as ocorrências onde o indivíduo persegue uma atividade para atingir um resultado ou satisfazer uma condição exterior à atividade. Por outro lado, um indivíduo está intrinsecamente motivado quando persegue uma atividade devido à experiência da atividade em si, sendo a realização da atividade o "resultado" que o indivíduo procura.

Todos os sistemas de jogos se baseiam em esquemas de reforço de razão variável, embora seus efeitos sejam geralmente atribuídos a sentimentos. Afirma-se frequentemente, por exemplo, que as pessoas jogam por excitação, mas este é claramente um produto colateral. Xu (2012) afirma que a gamificação está calçada no processo de mutação pelo qual a sociedade está passando, baseando-se no prazer e satisfação, em oposição à sua prioridade inicial de sobrevivência. Huizinga (1980) descreve que a denominação "homo sapiens" perdeu força quando passamos a perceber que não somos tão racionais quanto imaginávamos. Propondo a expressão "Homo Ludens" como uma alternativa para abranger os aspectos tão importantes quanto o raciocínio e a fabricação de objetos: o jogo. Para ele é através do jogo e pelo jogo que a civilização surge e se desenvolve.

\section{CONSIDERACִÕES FINAIS}

Atividades que utilizam o processo de gamificação de forma consciente tendem a conduzir o usuário de forma gradual e progressiva em um processo que o leve a realizar a tarefa desejada de forma agradável e divertida. Utilizando metáforas, desafios e feedbacks este processo estimula um determinado comportamento e oferece recompensas que podem compreender "bens" tangíveis tais como pontuação, níveis, distintivos, troféus ou não-tangíveis onde o principal deles é o status.

Desta forma, os sistemas gamificados podem ser utilizados como ferramentas importantes para os processos que objetivam influenciar de forma positiva o comportamento do usuário. Seja no estímulo ao aprendizado participativo dentro da educação, onde tarefas consideradas tediosas ou mesmo exaustivas ganham um componente motivacional, ou mesmo no reforço de condutas éticas, como reciclagem do lixo ou a obediência às normas de trânsito, por exemplo, a gamificação se apresenta como um leque de possibilidades capaz de influenciar na mudança tanto da conduta individual como coletiva.

Atualmente é possível observar inúmeras ações sociais que se utilizam dos princípios behavioristas por meio de dinâmicas de jogos e, à medida que 
o tema se torna mais difundido e claro, estas ações ganham em qualidade e amplitude. A designer de games Jane McGonigal (2011) defende que os games tem potencial para mudar o mundo, uma vez que possuem grande poder de influenciar comportamentos e que uma parcela considerável da população dispende boa parte de seu tempo nesta prática. Para a autora, basta direcionar os games ou os sistemas gamificados às ações corretas e focar na solução dos problemas que afligem a sociedade e será possível observar uma mudança coletiva e veloz.

\section{REFERÊNCIAS}

AZARITE, Ricardo. Gamification: como gerar engajamento com jogos nas redes sociais. 2013. Disponível em: <http://ideas.scup.com/pt/files/ downloads/2013/05/gamification_-scupideas.pdf>. Acesso em: 11 jun. 2013.

BAKKES, Sander, TAN, Chek Tien, PISAN, Yusuf. Personalised gaming: a motivation and overview of literature. In: AUSTRALASIAN CONFERENCE ON INTERACTIVE ENTERTAINMENT: PLAYING THE SYSTEM, $8^{\text {th }}$ 2012, Nova lorque. Proceedings... New York: ACM, 2012.

BALLONE, Geraldo José. Alterações da atividade voluntária. Disponível em: <http://www.psiqweb.med.br/site/?area=NO/LerNoticia\&idNoticia=202>. Acesso em: 11 maio 2008.

CAILLOIS, Roger. The definition of play, the classification of games. In: SALEN, Katie, ZIMMERMAN, Eric. The game design reader: a rules of play anthology. London: MIT Press, 2006.

DETERDING, Sebastian, KHALED, Rilla, NACKE, Lennart E., DIXON, Dan. Gamification: toward a definition. 2011a. Disponível em: <gamification-research. org/wp-content/uploads/2011/04/02-Deterding-Khaled-Nacke-Dixon.pdf>. Acesso: jun. 2013.

DETERDING, Sebastian, SICART, Miguel, NACKE, Lennart E., O'HARA, Kenton, DIXON, Dan. Using game design elements in non-gaming contexts. 2011b. Disponível em: <http://gamification-research.org/wp-content/ uploads/2011/04/01-Deterding-Sicart-Nacke-OHara-Dixon.pdf>. Acesso em: 11 jun. 2013.

FRASCA, Gonzalo. Ludology meets narratology: similitude and differences between (video) games and narrative. 1999. Disponível em: <http://www.ludology.org/ articles/ludology.htm>. Acesso em 20 abr. 2013. 
GROH, Fabian. Gamification: state of the art definition and utilization. In: SEMINAR ON RESEARCH TRENDS IN MEDIA INFORMATICS, 4th, 2012, Ulm. Proceedings... Ulm: University Institute of Media Informatics, 2012. p. 39-46.

HUIZINGA, Johan. Homu ludens: jogo como elemento da cultura. Tradução de João Paulo Monteiro. São Paulo: Perspectiva, 1980.

$\mathrm{KOHN}$, Alfie. Punished by rewards: the trouble with gold stars, incentive plans, a's, praise and other bribes. Nova lorque: Houghton Mifflin Company, 1999.

McGONIGAL, Jane. Reality is broken: why games make us better and how they can change the world. Penguin Press, New York, 2011.

MOORE, Michael. Basics of Game Design. London: CRC Press, 2001.

OLIVER, Richard L. Satisfaction: a behavioral perspective on the consumer. 2. ed. New York, ME Sharpe Inc., 2010.

SRIDHARAN, Mithun, HRISHIKESH, Aditya, RAJ, Lena Sunali. An academic analysis of gamification. 2012. Disponível em: <http://uxmag.com/resources/ an-academic-analysis-of-gamification>. Acesso em: 24 jun. 2013.

SKINNER, Burrhus Frederic. Sobre o behaviorismo. São Paulo: Cultrix, 1982.

ZILIO, Diego. A natureza comportamental da mente: behaviorismo radical e filosofia da mente. São Paulo: Cultura Acadêmica, 2010.

$X U$, Yongwen. Literature review on web application gamification and analytics. 2012. Disponível em: <https://csdl-techreports.googlecode.com/svn/trunk/ techreports/2011/11-05/11-05.pdf>. Acesso: 11 jun. 2013. 